\title{
STRUCTURE AND BONDING IN FIRST-ROW TRANSITION METAL DICARBIDE CATIONS $\mathrm{MC}_{2}^{+}$
}

\section{Supporting Information}

\author{
Víctor M. Rayón*, Pilar Redondo, Carmen Barrientos, and Antonio Largo* \\ Departamento de Química Física y Química Inorgánica \\ Facultad de Ciencias \\ Universidad de Valladolid \\ 47005 Valladolid \\ Spain
}

* Authors to whom correspondence should be addressed.

e-mail: vmrr@qf.uva.es, alargo@qf.uva.es

Fax: 34-983-423013 


\section{Complete Reference 45:}

Frisch, M. J., Trucks, G. W., Schlegel, H. B.; Scuseria, G. E.; Robb, M. A.; Cheeseman, J. R.; Zakrzewski, V. G.; Montgomery Jr. J. A.; Stratmann, R. E.; Burant, J. C.; Dapprich, S.; Millan, J. M.; Daniels, A. D.; Kudin, K. N.; Strain, M. C.;. Farkas, O.; Tomasi, J.; Barone, V.; Cossi, M.; Cammi, R.; Mennucci, B.; Pomelly, C.; Adamo, C.; Clifford, S.; Ochterski, J.; Petersson, G. A.; Ayala, P. Y.; Cui, Q.; Morokuma, K.; Malick, D. K.; Rabuck, A. D.; Raghavachari, K.; Foresman, J. B.; Cioslowski, J.; Ortiz, J. V.; Baboul, A. G.; Stefanov, B. B.; Liu, G.; Liashenko, A.; Piskorz, P.; Komaromi, I.; Gomperts, R.; Martin, R. L.; Fox, D. J.; Keith, T.; Al-Laham, M. A.; Peng, C. Y.; Nanayakkara, A.; Gonzalez, C.; Challacombe, M.;

Gill, P. M. W.; Johnson, B.; Chen, W.; Wong, M. W.; Andres, J. L.; Gonzalez, C.; HeadGordon, M.; Replogle, E. S.; Pople, J. A. Gaussian 98, Gaussian Inc, Pittsburgh, PA, 1998.

\section{Complete Reference 46:}

MOLPRO, a package of ab initio programs designed by H.-J. Werner and P. J. Knowles, version 2002.1, R. D. Amos, A. Bernhardsson, A. Berning, P. Celani, D. L. Cooper, M. J. O. Deegan, A. J. Dobbyn, F. Eckert, C. Hampel, G. Hetzer, P. J. Knowles, T. Korona, R. Lindh, A. W. Lloyd, S. J. McNicholas, F. R. Manby, W. Meyer, M. E. Mura, A. Nicklass, P. Palmieri, R. Pitzer, G. Rauhut, M. Schütz, U. Schumann, H. Stoll, A. J. Stone, R. Tarroni, T. Thorsteinsson, and H.-J. Werner. 
Table S1: Harmonic vibrational frequencies $\left(\mathrm{cm}^{-1}\right)$ and IR intensities $\left(\mathrm{km} \mathrm{mol}^{-1}\right)$, in parentheses, for linear $\mathrm{MC}_{2}^{+}$species obtained with the B3LYP/6-311+G(3df) and QCISD/6-311+G(d) (second line) methods.

\begin{tabular}{ll}
\hline Linear $\mathrm{MC}_{2}^{+}$ & \multicolumn{1}{c}{ Vibrational frequencies (IR intensities) } \\
\hline $\mathrm{ScC}_{2}{ }^{+}\left({ }^{1} \Sigma^{+}\right)^{\mathrm{a}}$ & $106 \mathrm{i}(7.47), 646(41.70), 1800(587.32)$ \\
& $60 \mathrm{i}(18.08), 647(21.97), 1751(254.20)$ \\
$\mathrm{TiC}_{2}{ }^{+}\left({ }^{2} \Delta\right)$ & $56 \mathrm{i}(5.40), 624(17.46), 1776(520.1)$ \\
& $81(5.87), 391(1.19), 1719(290.86)$ \\
$\mathrm{VC}_{2}{ }^{+}\left({ }^{5} \Delta\right)$ & $154(2.50), 516(60.21), 1926(79.47)$ \\
& $48(32.46), 527(90.97), 1954(83.94)$ \\
$\mathrm{CrC}_{2}{ }^{+}\left({ }^{6} \Sigma^{+}\right)$ & $171(19.59), 538(51.11), 1941(90.29)$ \\
& $176(32.85), 543(74.90), 1975(92.68)$ \\
$\mathrm{MnC}_{2}{ }^{+}\left({ }^{7} \Sigma^{+}\right)$ & $167(29.30), 530(59.50), 1970(167.13)$ \\
& $155(42.62), 516(83.89), 1959(118.15)$ \\
$\mathrm{FeC}_{2}{ }^{+}\left({ }^{6} \Delta\right)$ & $176(26.96), 547(43.86), 1949(219.04)$ \\
& $176(40.58), 546(90.40), 1917(280.16)$ \\
$\mathrm{CoC}_{2}{ }^{+}\left({ }^{5} \Pi\right){ }^{\mathrm{b}}$ & $242 \mathrm{i}(-), 227(25.05), 521(5.06), 1951(225.77)$ \\
& $179(22.67), 207(36.50), 552(62.73), 1956$ \\
$\mathrm{NiC}_{2}{ }^{+}\left({ }^{4} \Sigma^{-}\right)$ & $384.40)$ \\
$\mathrm{CuC}_{2}{ }^{+}\left({ }^{3} \Pi\right)$ & $567 \mathrm{i}(-), 491(78.52), 1688(128.24)$ \\
& $90(0.28), 260(12.51), 438(2.37), 1829(12.01)$ \\
$\mathrm{ZnC}_{2}{ }^{+}\left({ }^{2} \Sigma^{+}\right)$ & $197(5.31), 278(14.52), 450(23.52), 1852(14.80)$ \\
& $73 \mathrm{i}(-), 543(11.90), 2130(35.46)$ \\
& $123(23.96), 553(20.48), 2075(33.19)$ \\
\hline
\end{tabular}

${ }^{a}$ QCISD values obtained with the LAN/-6+(d) basis set

${ }^{\mathrm{b}}$ Note that non-degenerate $\pi$ vibrational frequencies, corresponding to the two Renner-Teller components, are obtained in this case. 
Table S2: Harmonic vibrational frequencies $\left(\mathrm{cm}^{-1}\right)$ and IR intensities $\left(\mathrm{km} \mathrm{mol}^{-1}\right)$, in parentheses, for cyclic $\mathrm{MC}_{2}{ }^{+}$species obtained with the B3LYP/6-311+G(3df) and QCISD/6-311+G(d) (second line) methods.

\begin{tabular}{cc}
\hline Cyclic $\mathrm{MC}_{2}{ }^{+}$ & Vibrational frequencies (IR intensities) \\
\hline $\mathrm{ScC}_{2}{ }^{+}\left({ }^{1} \mathrm{~A}_{1}\right)$ & $473(19.24), 684(84.84), 1794(21.69)$ \\
& $454(7.21), 735(44.54), 1805(10.86)$ \\
$\mathrm{TiC}_{2}{ }^{+}\left({ }^{2} \mathrm{~A}_{2}\right)$ & $473(17.80), 720(53.00), 1777(19.50)$ \\
& $458(1.82), 573(23.61), 1780(5.74)$ \\
$\mathrm{VC}_{2}{ }^{+}\left({ }^{3} \mathrm{~B}_{1}\right)$ & $197(4.68), 854(24.02), 1653(7.01)$ \\
$\mathrm{CrC}_{2}{ }^{+}\left({ }^{4} \mathrm{~B}_{1}\right)$ & $320(37.19), 641(1722.80), 1599(190.58)$ \\
& $354(2.42), 402(39.32), 1640(34.32)$ \\
$\mathrm{MnC}_{2}{ }^{+}\left({ }^{5} \mathrm{~A}_{1}\right)$ & $199(6.65), 465(38.45), 1776(31.00)$ \\
& $264(10.17), 473(57.32), 1747(19.79)$ \\
$\mathrm{FeC}_{2}{ }^{+}\left({ }^{6} \mathrm{~A}_{1}\right)$ & $347(8.19), 434(13.57), 1724(34.90)$ \\
& $326(23.79), 506(36.97), 1748(13.81)$ \\
$\mathrm{CoC}_{2}{ }^{+}\left({ }^{5} \mathrm{~A}_{2}\right)$ & $390(2.73), 491(10.30), 1683(39.59)$ \\
& $387(6.24), 479(46.34), 1712(13.37)$ \\
$\mathrm{NiC}_{2}{ }^{+}\left({ }^{4} \mathrm{~A}_{2}\right)$ & $376(4.61), 498(7.77), 1665(58.90)$ \\
$\mathrm{CuC}_{2}{ }^{+}\left({ }^{3} \mathrm{~B}_{1}\right)$ & $396(7.91), 521(22.22), 1713(8.76)$ \\
& $222(2.62), 406(3.05), 1558(45.35)$ \\
$\mathrm{ZnC}_{2}{ }^{+}\left({ }^{2} \mathrm{~A}_{1}\right)$ & $280(1.85), 466(0.49), 1553(18.30)$ \\
& $245(8.73), 472(5.60), 1736(61.59)$ \\
&
\end{tabular}


Table S3: Leading CASSCF CI coefficients ( 0.10 cutoff) and absolute CASSCF energies (in hartree) for the ground electronic states of the linear and cyclic isomers $\mathrm{TMC}_{2}{ }^{+}$ (TM=Sc-Zn).

$\underline{\text { Linear Isomers }}$

$\mathrm{ScC}_{2}^{+}\left({ }^{1} \Sigma^{+}\right) \quad-835.275988$

$0.91\left|\{\operatorname{core}\} 8 \sigma^{2} 9 \sigma^{2} 3 \pi^{4} 10 \sigma^{2}\right\rangle-0.12\left(\left|8 \sigma^{2} 9 \sigma^{2} 3 \pi_{x}^{2} 4 \pi_{y}^{2} 10 \sigma^{2}\right\rangle+\left|8 \sigma^{2} 9 \sigma^{2} 3 \pi_{y}^{2} 4 \pi_{x}^{2} 10 \sigma^{2}\right\rangle\right)$

$\mathrm{TiC}_{2}^{+}\left({ }^{2} \Delta\right) \quad-923.893141$

$0.87\left|\{\operatorname{core}\} 8 \sigma^{2} 9 \sigma^{2} 3 \pi^{4} 10 \sigma^{2} 1 \delta^{1}\right\rangle-0.13\left(\left|8 \sigma^{2} 9 \sigma^{2} 3 \pi_{x}^{2} 4 \pi_{y}^{2} 10 \sigma^{2} 1 \delta^{1}\right\rangle+\left|8 \sigma^{2} 9 \sigma^{2} 3 \pi_{y}^{2} 4 \pi_{x}^{2} 10 \sigma^{2} 1 \delta^{1}\right\rangle\right)+$

$+0.11\left(\left|8 \sigma^{2} 9 \sigma^{2} 3 \pi_{x}^{\alpha} 4 \pi_{x}^{\beta} 3 \pi_{y}^{\beta} 4 \pi_{x}^{\alpha} 10 \sigma^{2} 1 \delta^{1}\right\rangle+\left|8 \sigma^{2} 9 \sigma^{2} 3 \pi_{x}^{\beta} 4 \pi_{x}^{\alpha} 3 \pi_{y}^{\alpha} 4 \pi_{x}^{\beta} 10 \sigma^{2} 1 \delta^{1}\right\rangle\right)-$

$-0.11\left|8 \sigma^{2} 9 \sigma^{2} 3 \pi_{x}^{\beta} 4 \pi_{x}^{\alpha} 3 \pi_{y}^{\beta} 4 \pi_{x}^{\alpha} 10 \sigma^{2} 1 \delta^{1}\right\rangle$

$\mathrm{VC}_{2}^{+}\left({ }^{5} \Delta\right) \quad-1018.349257$

$0.93\left|\{\operatorname{core}\} 8 \sigma^{2} 9 \sigma^{2} 3 \pi^{4} 4 \pi^{2} 10 \sigma^{1} 1 \delta^{1}\right\rangle-$

$-0.13\left(\left|8 \sigma^{2} 9 \sigma^{2} 3 \pi_{y}^{2} 4 \pi^{2} 5 \pi_{x}^{2} 10 \sigma^{1} 1 \delta^{1}\right\rangle+\left|8 \sigma^{2} 9 \sigma^{2} 3 \pi_{x}^{2} 4 \pi^{2} 5 \pi_{y}^{2} 10 \sigma^{1} 1 \delta^{1}\right\rangle\right)$

$\mathrm{CrC}_{2}^{+}\left({ }^{6} \Sigma^{+}\right) \quad-1118.763070$

$0.93\left|\{\operatorname{core}\} 8 \sigma^{2} 9 \sigma^{2} 3 \pi^{4} 4 \pi^{2} 10 \sigma^{1} 1 \delta^{2}\right\rangle-$

$-0.13\left(\left|8 \sigma^{2} 9 \sigma^{2} 3 \pi_{y}^{2} 4 \pi^{2} 5 \pi_{x}^{2} 10 \sigma^{1} 1 \delta^{2}\right\rangle+\left|8 \sigma^{2} 9 \sigma^{2} 3 \pi_{x}^{2} 4 \pi^{2} 5 \pi_{y}^{2} 10 \sigma^{1} 1 \delta^{2}\right\rangle\right)$

$\mathrm{MnC}_{2}{ }^{+}\left({ }^{7} \Sigma^{+}\right) \quad-1225.238195$

$0.93 \mid\{$ core $\left.\} 8 \sigma^{2} 9 \sigma^{2} 3 \pi^{4} 4 \pi^{2} 10 \sigma^{1} 1 \delta^{2} 11 \sigma^{1}\right\rangle-$

$-0.13\left(\left|8 \sigma^{2} 9 \sigma^{2} 3 \pi_{y}^{2} 4 \pi^{2} 5 \pi_{x}^{2} 10 \sigma^{1} 1 \delta^{2} 11 \sigma^{1}\right\rangle+\left|8 \sigma^{2} 9 \sigma^{2} 3 \pi_{x}^{2} 4 \pi^{2} 5 \pi_{y}^{2} 10 \sigma^{1} 1 \delta^{2} 11 \sigma^{1}\right\rangle\right)$

$\mathrm{FeC}_{2}{ }^{+}\left({ }^{6} \Delta\right) \quad-1337.808687$

$0.94 \mid\{$ core $\left.\} 8 \sigma^{2} 9 \sigma^{2} 3 \pi^{4} 4 \pi^{2} 10 \sigma^{1} 1 \delta^{3} 11 \sigma^{1}\right\rangle-$

$-0.13\left(\left|8 \sigma^{2} 9 \sigma^{2} 3 \pi_{y}^{2} 4 \pi^{2} 5 \pi_{x}^{2} 10 \sigma^{1} 1 \delta^{3} 11 \sigma^{1}\right\rangle+\left|8 \sigma^{2} 9 \sigma^{2} 3 \pi_{x}^{2} 4 \pi^{2} 5 \pi_{y}^{2} 10 \sigma^{1} 1 \delta^{3} 11 \sigma^{1}\right\rangle\right)$

$\mathrm{CoC}_{2}+\left({ }^{5} \Pi\right)$ (State-averaged (SA) CASSCF of the ${ }^{5} \Pi$ and ${ }^{5} \Delta$ states) $\quad-1456.735501$ 
$0.55 \mid\{$ core $\left.\} 8 \sigma^{2} 9 \sigma^{2} 3 \pi^{4} 4 \pi^{3} 10 \sigma^{1} 1 \delta^{3} 11 \sigma^{1}\right\rangle-0.52 \mid\{$ core $\left.\} 8 \sigma^{2} 9 \sigma^{2} 3 \pi^{4} 4 \pi^{3} 10 \sigma^{2} 1 \delta^{2} 11 \sigma^{1}\right\rangle$

$\mathrm{NiC}_{2}^{+}\left({ }^{4} \Sigma^{-}\right) \quad-1582.164380$

$0.87 \mid\{$ core $\left.\} 8 \sigma^{2} 9 \sigma^{2} 3 \pi^{4} 4 \pi^{2} 10 \sigma^{2} 1 \delta^{4} 11 \sigma^{1}\right\rangle+0.34 \mid\{$ core $\left.\} 8 \sigma^{2} 9 \sigma^{2} 3 \pi^{4} 4 \pi^{4} 10 \sigma^{2} 1 \delta^{2} 11 \sigma^{1}\right\rangle-$

$\left.-0.12\left(8 \sigma^{2} 9 \sigma^{2} 3 \pi_{y}^{2} 4 \pi^{2} 5 \pi_{x}^{2} 10 \sigma^{2} 1 \delta^{4} 11 \sigma^{1}\right\rangle+\left|8 \sigma^{2} 9 \sigma^{2} 3 \pi_{x}^{2} 4 \pi^{2} 5 \pi_{y}^{2} 10 \sigma^{2} 1 \delta^{4} 11 \sigma^{1}\right\rangle\right)$

$\mathrm{CuC}_{2}^{+}\left({ }^{3} \Pi\right) \quad-1714.239417$

$0.95 \mid\{$ core $\left.\} 8 \sigma^{2} 9 \sigma^{2} 3 \pi^{4} 4 \pi^{3} 10 \sigma^{2} 1 \delta^{4} 11 \sigma^{1}\right\rangle-0.15 \mid\{$ core $\left.\} 8 \sigma^{2} 9 \sigma^{2} 3 \pi^{4} 4 \pi_{x}^{\alpha} 4 \pi_{y}^{0} 5 \pi_{y}^{2} 10 \sigma^{2} 1 \delta^{4} 11 \sigma^{1}\right\rangle-$

$-0.10\left|\{\operatorname{core}\} 8 \sigma^{2} 9 \sigma^{2} 3 \pi^{4} 4 \pi_{x}^{0} 4 \pi_{y}^{\alpha} 5 \pi_{x}^{\alpha} 5 \pi_{y}^{\beta} 10 \sigma^{2} 1 \delta^{4} 11 \sigma^{1}\right\rangle$

$\left.\mathrm{ZnC}_{2}{ }^{+}{ }^{2} \Sigma^{+}\right) \quad-1853.088097$

$0.94\left|\{\operatorname{core}\} 8 \sigma^{2} 9 \sigma^{2} 3 \pi^{4} 4 \pi^{4} 10 \sigma^{2} 1 \delta^{4} 11 \sigma^{1}\right\rangle-$

$-0.13\left(\mid\{\right.$ core $\left.\left.\} 8 \sigma^{2} 9 \sigma^{2} 3 \pi^{4} 4 \pi_{x}^{2} 5 \pi_{y}^{2} 10 \sigma^{2} 1 \delta^{4} 11 \sigma^{1}\right\rangle+\left|\{\operatorname{core}\} 8 \sigma^{2} 9 \sigma^{2} 3 \pi^{4} 4 \pi_{y}^{2} 5 \pi_{x}^{2} 10 \sigma^{2} 1 \delta^{4} 11 \sigma^{1}\right\rangle\right)$

\section{Cyclic Isomers}

$\mathrm{ScC}_{2}{ }^{+}\left({ }^{1} \mathrm{~A}_{1}\right) \quad-835.302513$

$0.92 \mid\{$ core $\left.\} 7 a_{1}^{2} 8 a_{1}^{2} 9 a_{1}^{2} 3 b_{1}^{2} 4 b_{2}^{2}\right\rangle-0.11 \mid\{$ core $\left.\} 7 a_{1}^{2} 8 a_{1}^{2} 10 a_{1}^{2} 3 b_{1}^{2} 4 b_{2}^{2}\right\rangle-0.10 \mid\{$ core $\left.\} 7 a_{1}^{2} 8 a_{1}^{2} 3 b_{1}^{2} 4 b_{2}^{2} 5 b_{2}^{2}\right\rangle$

$\mathrm{TiC}_{2}{ }^{+}\left({ }^{2} \mathrm{~A}_{2}\right) \quad-923.926804$

$0.89 \mid\{$ core $\left.\} 7 a_{1}^{2} 8 a_{1}^{2} 9 a_{1}^{2} 3 b_{1}^{2} 4 b_{2}^{2} 1 a_{2}^{1}\right\rangle-0.18 \mid\{$ core $\left.\} 7 a_{1}^{2} 8 a_{1}^{2} 10 a_{1}^{2} 3 b_{1}^{2} 4 b_{2}^{2} 1 a_{2}^{1}\right\rangle+$

$+0.12 \mid\{$ core $\left.\} 7 a_{1}^{2} 8 a_{1}^{2} 9 a_{1}^{\alpha} 10 a_{1}^{\alpha} 3 b_{1}^{2} 4 b_{2}^{2} 1 a_{2}^{\beta}\right\rangle$

$\mathrm{VC}_{2}^{+}\left({ }^{3} \mathrm{~B}_{1}\right) \quad-1018.338415$

$0.87 \mid\{$ core $\left.\} 7 a_{1}^{2} 8 a_{1}^{2} 9 a_{1}^{2} 3 b_{1}^{2} 4 b_{2}^{2} 1 a_{2}^{1} 5 b_{2}^{1}\right\rangle-0.17 \mid\{$ core $\left.\} 7 a_{1}^{2} 8 a_{1}^{2} 10 a_{1}^{2} 3 b_{1}^{2} 4 b_{2}^{2} 1 a_{2}^{1} 5 b_{2}^{1}\right\rangle-$

$-0.11 \mid\{$ core $\left.\} 7 a_{1}^{2} 8 a_{1}^{2} 9 a_{1}^{\alpha} 10 a_{1}^{\alpha} 3 b_{1}^{2} 4 b_{2}^{2} 1 a_{2}^{\beta} 5 b_{2}^{\alpha}\right\rangle-0.10 \mid\{$ core $\left.\} a_{1}^{2} 8 a_{1}^{2} 9 a_{1}^{2} 4 b_{1}^{2} 4 b_{2}^{2} 1 a_{2}^{1} 5 b_{2}^{1}\right\rangle$

$\mathrm{CrC}_{2}{ }^{+}\left({ }^{4} \mathrm{~B}_{1}\right) \quad-1118.734439$

$0.75 \mid\{$ core $\left.\} 7 a_{1}^{2} 8 a_{1}^{2} 9 a_{1}^{2} 3 b_{1}^{2} 4 b_{2}^{2} 1 a_{2}^{1} 5 b_{2}^{1} 10 a_{1}^{1}\right\rangle-0.28 \mid\{$ core $\left.\} 7 a_{1}^{2} 8 a_{1}^{2} 9 a_{1}^{0} 3 b_{1}^{2} 4 b_{2}^{2} 1 a_{2}^{1} 5 b_{2}^{1} 10 a_{1}^{1} 11 a_{1}^{2}\right\rangle-$ 
$-0.24\{$ core $\left.\} 7 a_{1}^{2} 8 a_{1}^{2} 9 a_{1}^{\beta} 3 b_{1}^{2} 4 b_{2}^{2} 1 a_{2}^{1} 5 b_{2}^{1} 10 a_{1}^{\alpha} 11 a_{1}^{\alpha}\right\rangle+0.16 \mid\{$ core $\left.\} 7 a_{1}^{2} 8 a_{1}^{2} 9 a_{1}^{\alpha} 3 b_{1}^{2} 4 b_{2}^{2} 1 a_{2}^{\beta} 5 b_{2}^{1} 10 a_{1}^{\alpha} 11 a_{1}^{\alpha}\right\rangle+$ $+0.16 \mid\{$ core $\left.\} 7 a_{1}^{2} 8 a_{1}^{2} 9 a_{1}^{\alpha} 3 b_{1}^{2} 4 b_{2}^{2} 1 a_{2}^{1} 5 b_{2}^{1} 10 a_{1}^{\beta} 11 a_{1}^{\alpha}\right\rangle-0.14 \mid\{$ core $\left.\} 7 a_{1}^{2} 8 a_{1}^{2} 9 a_{1}^{\alpha} 3 b_{1}^{2} 4 b_{2}^{2} 1 a_{2}^{1} 5 b_{2}^{1} 10 a_{1}^{\alpha} 11 a_{1}^{\beta}\right\rangle-$ $-0.13 \mid\{$ core $\left.\} 7 a_{1}^{2} 8 a_{1}^{2} 9 a_{1}^{2} 3 b_{1}^{\beta} 4 b_{1}^{\alpha} 4 b_{2}^{2} 1 a_{2}^{1} 5 b_{2}^{1} 10 a_{1}^{1}\right\rangle+0.11 \mid\{$ core $\left.\} 7 a_{1}^{2} 8 a_{1}^{2} 9 a_{1}^{\alpha} 3 b_{1}^{\beta} 4 b_{1}^{\alpha} 4 b_{2}^{2} 1 a_{2}^{1} 5 b_{2}^{1} 10 a_{1}^{\alpha} 11 a_{1}^{\beta}\right\rangle+$ $+0.10 \mid\{$ core $\left.\} 7 a_{1}^{2} 8 a_{1}^{2} 9 a_{1}^{\beta} 3 b_{1}^{\alpha} 4 b_{1}^{\beta} 4 b_{2}^{2} 1 a_{2}^{1} 5 b_{2}^{1} 10 a_{1}^{\alpha} 11 a_{1}^{\alpha}\right\rangle$

$\mathrm{MnC}_{2}{ }^{+}\left({ }^{5} \mathrm{~A}_{1}\right)-1225.234559$

$0.61 \mid\{$ core $\left.\} 7 a_{1}^{2} 8 a_{1}^{2} 9 a_{1}^{2} 3 b_{1}^{2} 4 b_{2}^{2} 1 a_{2}^{1} 5 b_{2}^{1} 10 a_{1}^{1} 4 b_{1}^{1}\right\rangle-0.43 \mid\{$ core $\left.\} 7 a_{1}^{2} 8 a_{1}^{2} 9 a_{1}^{0} 3 b_{1}^{2} 4 b_{2}^{2} 1 a_{2}^{1} 5 b_{2}^{1} 10 a_{1}^{1} 11 a_{1}^{2}\right\rangle-$

$-0.35 \mid\{$ core $\left.\} 7 a_{1}^{2} 8 a_{1}^{2} 9 a_{1}^{\beta} 3 b_{1}^{2} 4 b_{2}^{2} 1 a_{2}^{1} 5 b_{2}^{1} 10 a_{1}^{\alpha} 4 b_{1}^{1} 11 a_{1}^{\alpha}\right\rangle-0.29 \mid\{$ core $\left.\} 7 a_{1}^{2} 8 a_{1}^{2} 9 a_{1}^{\alpha} 3 b_{1}^{2} 4 b_{2}^{2} 1 a_{2}^{1} 5 b_{2}^{1} 10 a_{1}^{\alpha} 4 b_{1}^{1} 11 a_{1}^{\beta}\right\rangle$ $+0.17 \mid\{$ core $\left.\} 7 a_{1}^{2} 8 a_{1}^{2} 9 a_{1}^{\alpha} 3 b_{1}^{2} 4 b_{2}^{2} 1 a_{2}^{\beta} 5 b_{2}^{1} 10 a_{1}^{\alpha} 4 b_{1}^{1} 11 a_{1}^{\alpha}\right\rangle-0.17 \mid\{$ core $\} 7 a_{1}^{2} 8 a_{1}^{2} 9 a_{1}^{\alpha} 3 b_{1}^{2} 4 b_{2}^{2} 1 a_{2}^{1} 5 b_{2}^{1} 10 a_{1}^{\beta} 4 b_{1}^{1} 11 a_{1}^{\alpha}$, $+0.16 \mid\{$ core $\left.\} 7 a_{1}^{2} 8 a_{1}^{2} 9 a_{1}^{\alpha} 3 b_{1}^{2} 4 b_{2}^{2} 1 a_{2}^{1} 5 b_{2}^{1} 10 a_{1}^{\alpha} 4 b_{1}^{\beta} 11 a_{1}^{\alpha}\right\rangle+0.14 \mid\{$ core $\} 7 a_{1}^{2} 8 a_{1}^{2} 9 a_{1}^{\alpha} 3 b_{1}^{2} 4 b_{2}^{2} 1 a_{2}^{1} 5 b_{2}^{\beta} 10 a_{1}^{\alpha} 4 b_{1}^{1} 11 a_{1}^{\alpha}$

$\mathrm{FeC}_{2}{ }^{+}\left({ }^{6} \mathrm{~A}_{1}\right) \quad-1337.796800$

$0.93 \mid\{$ core $\left.\} 7 a_{1}^{2} 8 a_{1}^{2} 9 a_{1}^{2} 3 b_{1}^{2} 4 b_{2}^{2} 1 a_{2}^{1} 5 b_{2}^{1} 10 a_{1}^{1} 4 b_{1}^{1} 11 a_{1}^{1}\right\rangle-0.12 \mid\{$ core $\} 7 a_{1}^{2} 8 a_{1}^{2} 9 a_{1}^{2} 3 b_{1}^{0} 4 b_{2}^{2} 1 a_{2}^{1} 5 b_{2}^{1} 10 a_{1}^{1} 4 b_{1}^{1} 11 a_{1}^{1} 2 a$;

$\mathrm{CoC}_{2}^{+}\left({ }^{5} \mathrm{~A}_{2}\right) \quad-1456.733622$

$0.92 \mid\{$ core $\left.\} 7 a_{1}^{2} 8 a_{1}^{2} 9 a_{1}^{2} 3 b_{1}^{2} 4 b_{2}^{2} 1 a_{2}^{2} 5 b_{2}^{1} 10 a_{1}^{1} 4 b_{1}^{1} 11 a_{1}^{1}\right\rangle-0.12 \mid\{$ core $\left.\} 7 a_{1}^{2} 8 a_{1}^{2} 9 a_{1}^{2} 3 b_{1}^{1} 4 b_{2}^{2} 1 a_{2}^{2} 5 b_{2}^{1} 10 a_{1}^{2} 4 b_{1}^{2} 11 a_{1}^{1}\right\rangle-$ $-0.11 \mid\{$ core $\left.\} 7 a_{1}^{2} 8 a_{1}^{2} 9 a_{1}^{2} 3 b_{1}^{0} 4 b_{2}^{2} 1 a_{2}^{2} 5 b_{2}^{1} 10 a_{1}^{1} 4 b_{1}^{1} 11 a_{1}^{1} 2 a_{2}^{2}\right\rangle$

$\mathrm{NiC}_{2}{ }^{+}\left({ }^{4} \mathrm{~A}_{2}\right) \quad-1582.158614$

$0.88\{$ core $\left.\} 7 a_{1}^{2} 8 a_{1}^{2} 9 a_{1}^{2} 3 b_{1}^{2} 4 b_{2}^{2} 1 a_{2}^{2} 5 b_{2}^{1} 10 a_{1}^{2} 4 b_{1}^{1} 11 a_{1}^{1}\right\rangle+0.31 \mid\{$ core $\left.\} a_{1}^{2} 8 a_{1}^{2} 9 a_{1}^{2} 3 b_{1}^{2} 4 b_{2}^{2} 1 a_{2}^{1} 5 b_{2}^{2} 10 a_{1}^{1} 4 b_{1}^{1} 11 a_{1}^{1}\right\rangle-$ $-0.11 \mid\{$ core $\left.\} 7 a_{1}^{2} 8 a_{1}^{2} 9 a_{1}^{2} 3 b_{1}^{0} 4 b_{2}^{2} 1 a_{2}^{2} 5 b_{2}^{1} 10 a_{1}^{2} 4 b_{1}^{1} 11 a_{1}^{1} 2 a_{2}^{2}\right\rangle$

$\mathrm{CuC}_{2}^{+}\left({ }^{3} \mathrm{~B}_{1}\right)-1714.228362$

$0.94 \mid\{$ core $\left.\} 7 a_{1}^{2} 8 a_{1}^{2} 9 a_{1}^{2} 3 b_{1}^{2} 4 b_{2}^{2} 1 a_{2}^{2} 5 b_{2}^{2} 10 a_{1}^{2} 4 b_{1}^{1} 11 a_{1}^{1}\right\rangle+0.17 \mid\{$ core $\left.\} 7 a_{1}^{2} 8 a_{1}^{2} 9 a_{1}^{2} 3 b_{1}^{2} 4 b_{2}^{2} 1 a_{2}^{2} 5 b_{2}^{1} 10 a_{1}^{2} 4 b_{1}^{0} 11 a_{1}^{2} 2 a_{2}^{1}\right\}+$ $+0.12 \mid\{$ core $\left.\} 7 a_{1}^{2} 8 a_{1}^{2} 9 a_{1}^{2} 3 b_{1}^{2} 4 b_{2}^{2} 1 a_{2}^{2} 5 b_{2}^{\alpha} 10 a_{1}^{\beta} 4 b_{1}^{1} 11 a_{1}^{1} 6 b_{2}^{\alpha}\right\rangle-0.11 \mid\{$ core $\} 7 a_{1}^{2} 8 a_{1}^{2} 9 a_{1}^{2} 3 b_{1}^{2} 4 b_{2}^{2} 1 a_{2}^{2} 5 b_{2}^{2} 10 a_{1}^{0} 4 b_{1}^{1} 11 a_{1}^{1} 6 i$

$\mathrm{ZnC}_{2}{ }^{+}\left({ }^{2} \mathrm{~A}_{1}\right) \quad-1853.074498$ 
$0.94 \mid\{$ core $\left.\} 7 a_{1}^{2} 8 a_{1}^{2} 9 a_{1}^{2} 3 b_{1}^{2} 4 b_{2}^{2} 1 a_{2}^{2} 5 b_{2}^{2} 10 a_{1}^{2} 4 b_{1}^{2} 11 a_{1}^{1}\right\rangle-0.13 \mid\{$ core $\left.\} 7 a_{1}^{2} 8 a_{1}^{2} 9 a_{1}^{2} 3 b_{1}^{2} 4 b_{2}^{2} 1 a_{2}^{2} 5 b_{2}^{2} 10 a_{1}^{2} 4 b_{1}^{0} 11 a_{1}^{1} 2 a_{2}^{2}\right\rangle-$ $-0.10 \mid\{$ core $\left.\} 7 a_{1}^{2} 8 a_{1}^{2} 9 a_{1}^{2} 3 b_{1}^{2} 4 b_{2}^{2} 1 a_{2}^{2} 5 b_{2}^{\alpha} 10 a_{1}^{2} 4 b_{1}^{\beta} 11 a_{1}^{2} 2 a_{2}^{\alpha}\right\rangle-0.10 \mid\{$ core $\} 7 a_{1}^{2} 8 a_{1}^{2} 9 a_{1}^{2} 3 b_{1}^{2} 4 b_{2}^{2} 1 a_{2}^{2} 5 b_{2}^{\alpha} 10 a_{1}^{2} 4 b_{1}^{2} 11 a_{1}^{\beta}$ 